\title{
THE HARVARD CUP MAN-VERSUS-MACHINE CHESS CHALLENGE
}

\author{
Christopher Chabris \\ Cambridge, MA / USA
}

In the summer of 1989 Dan Edelman, a FIDE Master and then president of the Harvard University Chess Club, was planning a visit by World Champion Garry Kasparov to the college. Kasparov would be traveling to Cambridge from New York, where he was scheduled to play the subsequently famous two-game match against Deep Thought. I was helping Dan to organize the visit, and we wanted to stage a man-against-machine confrontation that would be different from the head-on match format but also intriguing for the public and the press. We were unaware of the AEGON tournaments at the time (that event was just evolving into its mature state), but we decided to adopt a similar formula: to pit teams of four computers and four American grandmasters against each other in the Scheveningen format. Each human would play each computer once, but no humans or computers would play each other.

After a lot of last-minute faxing and telephoning the Harvard Chess Festival was set to begin. Kasparov arrived in the afternoon of Friday, 27 October 1989, spoke at the Russian Research Center, and on the next day shut out eight opponents (seven humans plus the Sargon IV program) in one of his trademark clock simultaneous displays before an audience of about 800. That evening he analyzed some positions with Deep Thought at a party in his honor, and at noon on Sunday the $29^{\text {th }}$ he came to Harvard's Memorial Hall, built to honor the university's Civil War dead, to open officially what we had dubbed the First Harvard Cup. In brief remarks he predicted victory for the grandmasters, but warned that he was always ready to defend humanity if necessary. As it turned out he was not needed that day: the human team, consisting of GMs Lev Alburt, Maxim Dlugy, Boris Gulko, and Michael Rohde won by a score of 14.5-1.5 against Chiptest, Deep Thought, Hitech, and Mephisto's Portorose (a unit with a Motorola 68020 processor running at $12 \mathrm{MHz}$ ).

George Mirijanian was the arbiter, IM Danny Kopec provided commentary, and there was a brief public discussion after the games were over. The sponsors included IBM and the American Chess Foundation (ACF). The games were played at G/30, the "active" time limit, and the grandmasters received appearance fees as well as prizes of $\$ 500$ and $\$ 300$ to the top two scorers. The top human and computer participants received invitations to the next edition as well as their names engraved on a trophy. Gulko and Rohde at 4-0 shared the prize money and were both invited to return, as was Deep Thought (whose developers later declined on its behalf).

\begin{tabular}{|l|llll|l|}
\hline & Deep Thought & $\begin{array}{l}\text { Mephisto } \\
\text { Portorose }\end{array}$ & Chiptest & Hitech & TOTAL \\
\hline Boris Gulko & $1-0$ & $1-0$ & $1-0$ & $1-0$ & 4.0 \\
Michael Rohde & $1-0$ & $1-0$ & $1-0$ & $1-0$ & 4.0 \\
Lev Alburt & $1-0$ & $1 / 2$ & $1-0$ & $1-0$ & 3.5 \\
Maxim Dlugy & $0-1$ & $1-0$ & $1-0$ & $1-0$ & 3.0 \\
\hline TOTAL & 1.0 & 0.5 & 0.0 & 0.0 & \\
\hline
\end{tabular}

Table 1: Results of the First Harvard Cup, 29 October 1989.

The game below was the first to finish in the First Harvard Cup. After Gulko's surprisingly easy demolition of Deep Thought, the audience gave Harvard's former Grandmaster in Residence a standing ovation while the other games were still in progress. 
Boris Gulko - Deep Thought (NicKey RE 22.2)

First Harvard Cup, Round 1

1. Nf3 d5 2. g3 Nf6 3. Bg2 c6 4. d3 Nbd7 5. 0-0 e5 6. Nc3 d4 7. Nb1 Bd6 8. e4 Nc5 9. Nbd2 b5 10. Nxd4 exd4 11. e5 Bb7 12. exd6 Qxd6 13. Re1+ Ne6 14. a4 a6 15. Ne4 Nxe4 16. Bxe4 0-0 17. Qh5 g6 18. Qh4 Qb4 19. Bh6 Rfb8 20. Bxg6 fxg6 21. Qf6 Rf8 22. Bxf8 Rxf8 23. Qxe6+ Kg7 24. Qe7+ Qxe7 25. Rxe7+ Rf7 26. Rae1 bxa4 27. R1e4 c5 28. R4e5 c4 29. dxc4 1-0

Alburt became the first Grandmaster to surrender a half point to a computer in the Harvard Cup series by letting a powerful bind deteriorate into an uncertain pawn race against Mephisto Portorose. But even after several inaccuracies, and allowing Mephisto to queen a Pawn, the two-time U.S. Champion retained enough tactical resources to force the draw.

\section{Lev Alburt - Mephisto Portorose (NicKey VO 22)}

First Harvard Cup, Round 2

1. d4 e6 2. g3 Nf6 3. Bg2 Nc6 4. Nf3 d5 5. 0-0 Be7 6. c4 0-0 7. Nc3 dxc4 8. e3 Na5 9. Qe2 c5 10. dxc5 Qc7 11. e4 Bxc5 12. Bg5 Ng4 13. e5 f6 14. exf6 gxf6 15. Bd2 Bd7 16. h3 Ne5 17. Nxe5 Qxe5 18. Qxe5 fxe5 19. Ne4 Bb6 20. Rad1 Ba4 21. Rc1 Bc6 22. Bc3 Rad8 23. Rc2 Rf5 24. Re2 h6 25. g4 Rf7 26. Bxe5 Rd3 27. Bc3 Kf8 28. Bxa5 Bxa5 29. Nc5 Bxg2 30. Kxg2 Rd5 31. Nxe6+ Kg8 32. f4 b5 33. Re4 Bb6 34. Rfe1 Rd2+ 35. R1e2 Rfd7 36. Kf1 Rd1+ 37. Re1 Ba5 38. Rxd1 Rxd1+ 39. Kf2 Bb6+ 40. Kg3 Rd2 41. a4 a6 42. Kh4 Rxb2 43. Kh5 c3 44. Kg6 Rb4 45. Nd4 Kf8 46. Ne6+ Kg8 47. Re5 c2 48. Rd5 c1/Q 49. Rd7 Qc3 50. Re7 Qc2+ 51. f5 Rxg4+ 52. hxg4 Qc6 53. Rg7+ Kh8 54. Rh7+ Kg8 55. Rg7+ Kh8 56. Rh7+ Kg8 0.5-0.5

Shortly after the above game finished, Dlugy gave up the first full point to a computer in Cup history, losing to Deep Thought. Ironically, Dlugy later worked as a consultant to the Deep Thought group at IBM. In the opening, Deep Thought allowed a pawn-fork trick in the center, just as it did against Gulko, and entered an endgame with a clear pawn deficit. But Dlugy slowly lost the thread of the position and was forced to surrender a piece, and with it the game.

\section{Deep Thought - Maxim Dlugy (NicKey SI 48.4.2)}

First Harvard Cup, Round 2

1. e4 c5 2. d4 cxd4 3. c3 dxc3 4. Nxc3 d6 5. Nf3 e6 6. Bc4 Be7 7. Bf4 Nc6 8. Rc1 Nf6 9. 0-0 0-0 10. Qd2 Nxe4 11. Nxe4 d5 12. Bd3 dxe4 13. Bxe4 Qxd2 14. Bxd2 Bd7 15. Rfe1 Rac8 16. Bf4 Bf6 17. Rcd1 Rfd8 18. b3 Be8 19. a4 h6 20. h3 g5 21. Be3 b6 22. Rxd8 Bxd8 23. Rc1 f5 24. Bd3 f4 25. Bd4 Bd7 26. Bb2 Na5 27. Rd1 Bc6 28. b4 Nb7 29. Ba6 Be7 30. Rc1 Bxb4 31. Ne5 Bc5 32. Nxc6 Rxc6 33. Bxb7 Rd6 34. Bc3 Kf7 35. Bf3 Kg6 36. Re1 Kf7 37. Bg4 Rc6 38. Bh5+ Ke7 39. Bg7 Bb4 40. Re4 Rc1+ 41. Kh2 Bd6 42. Bg4 f3+ 43. g3 Rf1 44. Bd4 Bc5 45. Bxe6 Kd6 46. Bc4 Rc1 47. Bb3 Rf1 48. g4 Bxd4 49. Rxd4+ Kc5 50. Rc4+ Kd6 51. Kg3 Rb1 52. Rc3 Ke5 53. Re3+ Kd4 54. Kxf3 a6 55. Bf7 b5 56. Re6 bxa4 57. Rxa6 Rb4 58. Rxh6 Kc3 59. Ke3 a3 60. Ra6 Kb2 61. Bd5 Rb5 62. Bc4 Rb8 63. Ra5 Rc8 64. Rb5+ Ka1 65. Kd3 Rf8 66. Rf5 Rh8 67. Re5 Rxh3+ 68. Kc2 Rh1 69. Ra5 Rh3 70. Bd5 Rd3 71. Kxd3 Kb2 72. Rb5+ Ka1 73. Kc2 1-0

After news of Kasparov's visit got out, we were contacted by Anatoly Karpov, who also wished to visit Harvard. We arranged a one-game match between the former world champion and Deep Thought in Memorial Hall on 2 February 1990. This game, which Karpov was lucky to win, was widely publicized, as was his analysis of the game, given the next day in a lecture prior to a large simultaneous exhibition.

Exhausted by all this organizing, we held no Harvard Cup in 1990, but in the next year we managed to revive the event in conjunction with another Harvard Chess Festival, this time in May, sponsored by Bankers Trust Company, Harvard alumnus Malcolm H. Wiener, and the ACF. Dlugy, Gulko, and Rohde returned and were joined by new grandmaster Patrick Wolff. On the evening of 3 May, once again in Memorial Hall, this time at G/25 "quickplay" or "rapid" tempo, after giving an outdoor exhibition for charity, they faced four computer opponents: Fidelity's Mach IV (with a $20 \mathrm{MHz} 68020$ processor), Mephisto's Lyon (12 MHz 68020), Larry Kaufman's Rex Chess, and Heuristic Software's prototype Alpha program. The latter two programs ran on PC clones based on the Intel 80486 processor, Rex Chess running at $25 \mathrm{MHz}$, Alpha at $33 \mathrm{MHz}$. Mirijanian returned as arbiter, and after the games Rohde analyzed his fantastic game against Alpha for the spectators. 
Despite the seemingly weaker computer team, the results of the Second Harvard Cup were startling: only 12-4 in favor of the human beings. Dlugy with 3.5-0.5 and Rohde and Wolff with 3-1 took the prize money, raised to $\$ 600$ first and $\$ 400$ second this year, and Dlugy was invited to play again. Alpha's impressive even score earned it the same invitation, as well as an entry (with Dlugy) on the trophy. Alpha's back-to-back victories against Wolff and Rohde represented both the first and second times that a microcomputer-based program ever defeated a Grandmaster in other than blitz (5-minute) or casual play.

\begin{tabular}{|l|llll|l|}
\hline & $\begin{array}{l}\text { Heuristic } \\
\text { Alpha }\end{array}$ & $\begin{array}{l}\text { Fidelity } \\
\text { Mach IV }\end{array}$ & Rex Chess & $\begin{array}{l}\text { Mephisto } \\
\text { Lyon }\end{array}$ & TOTAL \\
\hline Maxim Dlugy & $1-0$ & $1 / 2$ & $1-0$ & $1-0$ & 3.5 \\
Michael Rohde & $0-1$ & $1-0$ & $1-0$ & $1-0$ & 3.0 \\
Patrick Wolff & $0-1$ & $1-0$ & $1-0$ & $1-0$ & 3.0 \\
Boris Gulko & $1-0$ & $0-1$ & $1 / 2$ & $1-0$ & 2.5 \\
\hline TOTAL & 2.0 & 1.5 & 0.5 & 0.0 & \\
\hline
\end{tabular}

Table 2: Results of the Second Harvard Cup, 3 May 1991.

Heuristic Alpha versus Rohde, from the second Cup, was the most exciting game of all three Cups; the spectators were on the edges of their seats during the thrilling 15-minute king hunt at the end. Rohde overlooked Alpha's $21^{\text {st }}$ move, the type of oblique shot computers rarely miss, but he converted his loss of the exchange into a courageous sacrifice of a full Rook to drive Alpha's King all the way up the board. Alpha could have forced a draw more than once, beginning with $29 . \mathrm{Kg} 3$, but instead played consistently to win, as did Rohde. After 35. Kf6 Rohde's intuition finally gave out and he went astray. Although a lengthy post-mortem didn't discover it, ironically, another computer later found an incredible winning idea for Black: 35. ... Ba5! 36. Nb6 $\mathrm{Bc3}+37 . \mathrm{Kg} 5 \mathrm{Bd} 4$ !! 38. Qd1 (all other tries lead to mate) Qe5+ 39. Kh4 Qxh2+ 40. Kg4 Qxg2+ 41. Kf4 Qxf2+ 42. Qf3 Be5+ winning the Queen. Even so, Rohde could still have salvaged a draw later with 43. ... Bd6! followed by perpetual check with the Queen on $\mathrm{a} 8, \mathrm{~b} 8$, and $\mathrm{d} 8$.

\section{Heuristic Alpha - Michael Rohde}

(NicKey FR 1.4.12)

Second Harvard Cup, Round 2

1. e4 e6 2. d4 d5 3. exd5 exd5 4. Nf3 Bd6 5. Bd3 Ne7

6. 0-0 0-0 7. b3 Nbc6 8. c4 dxc4 9. bxc4 Nb4 10. Be2 c5 11. a3 Nbc6 12. d5 Ne5 13. Nc3 Bf5 14. Nh4 Re8 15. Re1 a6 16. Bg5 Qd7 17. Na4 f6 18. Nb6 Qd8 19. Nxa8 fxg5 20. Nxf5 Nxf5 21. Qb1 Nd4 22. Nb6 Nxe2+ 23. Rxe2 Nf3+ 24. Kf1 Rxe2 25. Kxe2 Qe8+ 26. Kxf3 g4+ 27. Kxg4 Qe2+ 28. Kh4 Be7+29. Kh3 Qh5+ 30. Kg3 Bh4+ 31. Kf4 Bg5+ 32. Ke4 Qe2+ 33 . Kf5 Bd2 34. Na4 g6+ 35. Kf6 (see Diagram 1) Qxf2+ 36. Ke7 Bg5+ 37. Kd6 Qf8+ 38. Kc7 b5 39. cxb5 Bf4+ 40. Kb6 Qd6+ 41. Ka7 Qb8+ 42. Kxa6 Qa8+ 43. Kb6 c4 44. Qe4 Qd8+ 45. Kc5 1-0 (time)

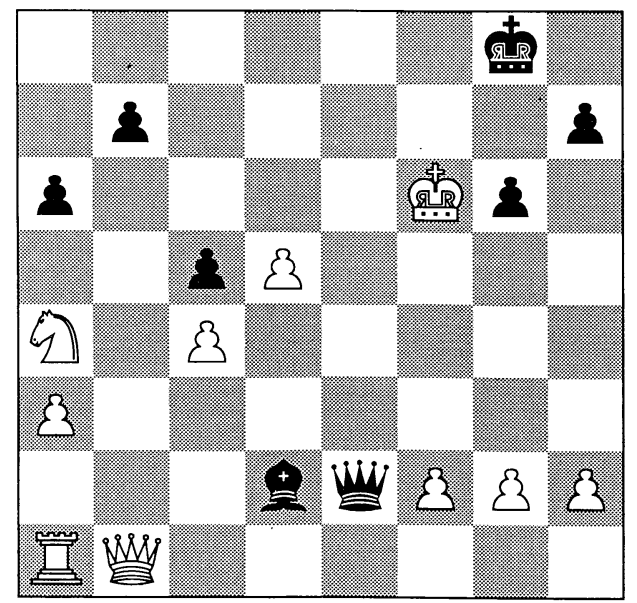

Diagram 1: Position after 35. Kf6. 
Alpha had also defeated Wolff in the first round, but Dlugy showed how to handle the program and snapped its 2-0 streak with this methodical positional victory, based on a monster c-file pin:

\section{Heuristic Alpha - Maxim Dlugy (NicKey SI 1.8)}

Second Harvard Cup, Round 3

1. e4 c5 2. Nf3 d6 3. Bb5+ Bd7 4. Bxd7+ Nxd7 5. 0-0 Ngf6 6. Qe2 e6 7. c3 Be7 8. d4 cxd4 9. cxd4 Nb6 10. Nc3 0-0 11. Bg5 d5 12. Bxf6 Bxf6 13. e5 Be7 14. Rac1 a6 15. Rfe1 Rc8 16. h3 Qd7 17. Rc2 Rc4 18. Rec1 Rfc8 19. a3 R8c7 20. Qd2 h6 21. Qd3 Qc8 22. Nd2 R4c6 23. Qg3 Bg5 24. f4 Be7 25. Kh1 Na4 26. Ndb1 b5 27. b4 a5 28. Qd3 axb4 29. axb4 Rc4 30. Kh2 Bxb4 31. Nxa4 Rxc2 32. Nb6 Rxc1 33. Nxc8 Rxc8 34. Qb3 R8c4 35. Qd3 g6 36. h4 h5 37. Qb3 Rxd4 38. Qb2 Rdc4 39. Nd2 R1c2 40. Nxc4 Rxb2 41. Nxb2 Bc3 42. Nd3 b4 43. Nc5 Bd4 44. Nb3 Bb6 45. Kg3 Bd8 46. Nd4 Kf8 47. Nb3 Ke8 48. Nd4 Kd7 49. Nb3 Kc6 50. Kh3 Kb5 51. Nd2 Ka4 52. g4 b3 53. gxh5 gxh5 54. Nb1 b2 55. Kg3 Kb3 56. Kf3 Kc2 57. Na3+ Kc1 58. f5 Be7 0-1

In 1992, the Malcolm H. Wiener Trophy, named in honor of the event's principal ongoing benefactor, was procured and engraved. The Third Harvard Cup was held in New York in conjunction with the first U.S. Chess Festival, and Intel Corporation and the Millburn Corporacion joined the list of sponsors for the first time. After the first day of the U.S. Quick Chess Championship (at G/10) in the afternoon of Saturday, 11 July 1992, the Cup began at about 7:30 p.m. at the auditorium of the Hunter College Campus Schools. This time five Grandmasters (Dlugy, Rohde, Wolff, and newcomers John Fedorowicz and Sergey Kudrin) faced Fidelity's Elite Premiere, Mephisto's RISC, ChessBase's Knightstalker (known as Fritz in Europe), The Software Toolworks's Chessmaster 3000, and Heuristic's Socrates, another unreleased protoype. The microcomputer programs again all ran on 80486 systems: Socrates at $50 \mathrm{MHz}$, Knightstalker at $33 \mathrm{MHz}$, and Chessmaster 3000 with the DX2 chip. Carol Jarecki served as arbiter, assisted by Sunil Weeramantry.

After a grueling evening, which included a discussion on how computers are affecting the game of chess (with panelists IM Larry Kaufman, GMs Robert Byrne and Joel Benjamin, and Drs. Feng-hsiung Hsu and Murray Campbell) and featured several disputes during the play, the human team won by an 18-7 margin. This represented a score of $28 \%$ for the computers, up from $25 \%$ in 1991 and $9 \%$ in 1989 . Rohde scored a clean 5-0 to collect $\$ 600$, an invitation, and his second entry on the trophy; Kudrin received $\$ 400$ for his $4-1$ effort. At 32 , Socrates became the first computer of any type ever to earn a positive score against a field of professional grandmasters in a single serious event, a fantastic achievement.

\begin{tabular}{|l|lllll|l|}
\hline & $\begin{array}{l}\text { Heuristic } \\
\text { Socrates }\end{array}$ & $\begin{array}{l}\text { Mephisto } \\
\text { RISC }\end{array}$ & $\begin{array}{l}\text { ChessBase } \\
\text { Knightstalker }\end{array}$ & $\begin{array}{l}\text { Chessmaster } \\
3000\end{array}$ & $\begin{array}{l}\text { Elite } \\
\text { Premiere }\end{array}$ & TOTAL \\
\hline Michael Rohde & $1-0$ & $1-0$ & $1-0$ & $1-0$ & $1-0$ & 5.0 \\
Sergey Kudrin & $1-0$ & $1-0$ & $0-1$ & $1-0$ & $1-0$ & 4.0 \\
Maxim Dlugy & $0-1$ & $0-1$ & $1-0$ & $1-0$ & $1-0$ & 3.0 \\
John Fedorowicz & $0-1$ & $0-1$ & $1-0$ & $1-0$ & $1-0$ & 3.0 \\
Patrick Wolff & $0-1$ & $1 / 2$ & $1-0$ & $1 / 2$ & $1-0$ & 3.0 \\
\hline TOTAL & 3.0 & 2.5 & 1.0 & 0.5 & 0.0 & \\
\hline
\end{tabular}

Table 3: Results of the Third Harvard Cup, 11 July 1992.

The Fedorowicz-Mephisto game was the most interesting of the third Cup. Mephisto RISC fell into what one of the players described as a "book trap" at move 8, but saw that 10... Qxd4 would lose to 11. Qa4+ Kd8 12. $\mathrm{Ba5}+\mathrm{b} 613$. Rd1 (if $11 \ldots \mathrm{Bd} 712$. Bxf7+, or $11 \ldots \mathrm{Qd} 712 . \mathrm{Bb} 5$ ). It then recovered nicely with threats that dislocated and tied down Fedorowicz's pieces. After 15... e4 it entered an endgame still down a piece for two Pawns, but with relentless pressure managed to convert this into a material imbalance of Bishop and two Pawns against Fedorowicz's Rook. With both sides running short of time, all of the other material on the board was exchanged until only these forces remained. As his flag hung with about 5 seconds to play, Fedorowicz claimed a draw because he had established a sort of "dynamic fortress" and could demonstrate the proper drawing technique. The arbiters conferred and decided to consider the claim after another ten moves had been played, 
but four moves later Mephisto won on time in what by then was a clearly winning position (W: Kd2, Rf2; B: $\mathrm{Ka} 1, \mathrm{Bb} 5, \mathrm{~Pb} 2)$. After its early blunder and a few inaccuracies by its opponent the computer played an impressive positional game that looked nothing like an artificial creation.

\begin{abstract}
John Fedorowicz - Mephisto RISC
(NicKey CK 3.4.2)

Third Hardvard Cup, Round 3

1. c4 c6 2. e4 d5 3. exd5 cxd5 4. d4 Nf6 5. Nc3 Nc6 6. Bg5 Qa5 7. Bd2 dxc4 8. Bxc4 Nxd4 9. Nb5 Qb6 10. Nxd4 e5 11. Nc2 Bc5 12. Qe2 Qxb2 13. Rc1 0-0 14. Qd3 Bg4 15. f3 e4 (see Diagram 2) 16. Qb3 Qxb3 17. Bxb3 exf3 18. gxf3 Rfe8+ 19. Kf1 Bf5 20. Re1 Rad8 21. Rxe8+ Rxe8 22. Ne1 Nh5 23. Bc4 Rc8 24. Bd3 Rd8 25. Ke2 Re8+ 26. Kd1 Rxe1+ 27. Kxe1 Bxd3 28. Ne2 Bc4 29. Nc1 f5 30. Kd1 Kf7 31. Kc2 Bd5 32. Rf1 Bd6 33. f4 Nf6 34. Nd3 Bc4 35. Rb1 b6 36. Ne5+ Bxe5 37. fxe5 Ne4 38. h4 Nxd2 39. Kxd2 Ke6 40. Re1 h6 41. Rg1 g5 42. hxg5 hxg5 43. Rxg5 Bxa2 44. Rg7 Kxe5 45. Rd7 Bd5 and eventually 0-1
\end{abstract}

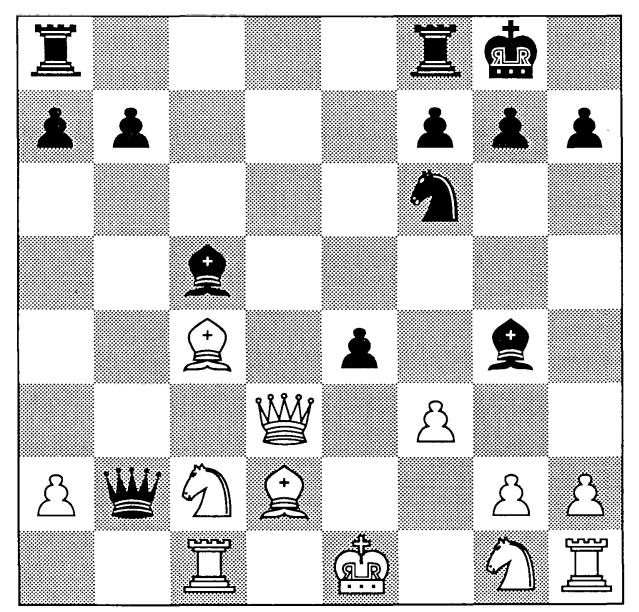

Diagram 2: Position after 15. ... e4.

Here, Kudrin falls into a pattern similar to that of Alpha-Rohde: unable to pull off an attack against ChessBase Knightstalker because of time pressure, he eventually runs out of tactics and loses.

\title{
Sergey Kudrin - ChessBase Knightstalker (NicKey RL 17.2.3)
}

Third Harvard Cup, Round 2

1. e4 e5 2. Nf3 Nc6 3. Bb5 a6 4. Ba4 Nf6 5. 0-0 Be7 6. Re1 b5 7. Bb3 0-0 8. h3 Bb7 9. d3 a5 10. a4 b4 11. Nbd2 d6 12. Nc4 Rb8 13. Ne3 h6 14. Nf5 Re8 15. Bxh6 gxh6 16. Nxh6+ Kg7 17. Nxf7 Qd7 18. Re3 Rf8 19. N7g5 d5 20. Nh4 Nxe4 21. dxe4 Bxg5 22. Rg3 Kh6 23. Nf5+ Rxf5 24. exf5 Ne7 25. Qe2 Bf4 26. Rg4 Nxf5 27. g3 Bg5 28. Rxg5 Kxg5 29. Qxe5 Re8 30. Qf4+ Kg6 31. Rd1 c5 32. c4 Nd4 33. Rxd4 cxd4 34. Bc2+ Kg7 35. Qxd4+ Kf7 36. Qf4+ Kg8 37. Kh2 dxc4 38. Qxc4+ Kg7 39. Bb3 Re1 40. Qg8+ Kh6 41. Qh8+ Kg6 42. Bc2+ Be4 43. Qg8+ Kf6 44. Qf8+ Qf7 45. Qh6+ Ke7 46. Qg5+ Kf8 47. Qd8+ Kg7 48. Qd4+ Qf6 49. Qd7+ Kf8 50. Qc8+ Ke7 51. Qc7+ Ke6 52. Bb3+ Kf5 53. Qd7+ Kg6 54. Qd2 Rh1+ 0-1

In 1989 it was obvious that Kasparov would defeat Deep Thought, but it was not so clear how a team of lesser Grandmasters would do against a team of top computers, and since matches between the World Champion and a computer do not happen often, the ongoing Harvard Cup series has provided a useful measurement of progress in computer chess. The Fourth Harvard Cup is being planned for later in 1993. As before, it will be open to commercial, prototype, and research machines and programs, who will face a squad of financially-motivated American Grandmasters. Among other improvements, it will have more explicit rules and eligibility policies to minimize disputes, and it may feature more participants on both teams than in the past. If you are interested in entering a program or a dedicated machine in the Harvard Cup, or in receiving more information about it, please contact the author of this article. Larry Kaufman has already predicted that if systems based on Intel's "Pentium" chip are available, the computers could show superiority over the Grandmasters for the first time ever - at least at the G/25 time limit! 